\title{
Trichotillomania in a young male complicated by tinea capitis associated with Cryptococcus laurentii and Candida parapsilosis
}

This article was published in the following Dove Press journal:

Clinical, Cosmetic and Investigational Dermatology

2 March 2013

Number of times this article has been viewed

Michael J Fellner

Department of Dermatology, New York Medical College, New York, NY, USA
Correspondence: Michael J Fellner Metropolitan Hospital, 50 East 89th Street, New York, NY 10128, USA Email freddagf@aol.com

\begin{abstract}
This is the first case report of tinea capitis associated with Cryptococcus laurentii as well as Candida parapsilosis in an eleven year old male which responded to treatments with griseofulvin, lamisil, as well as fluconazole only to show recurrent alopecia from an underlying problem of trichotillomania. When confronted with the biopsy diagnosis of trichotillomania, the family admitted knowledge of the hair pulling disorder for at least five years. It took three providers to finally solve the question of the recalcitrant alopecia.
\end{abstract}

Keywords: tinea capitis, trichotillomania, lamisil, fluconazole, griseofulvin, cryptococcus laurentii, candida parapsilosis

\section{Introduction}

Tinea capitis is a disease manifesting as various degrees of alopecia, usually in preadolescent children, although adults can be affected. It commonly affects the scalp, but eyebrows and eyelashes may be involved. ${ }^{1}$ The most common cause is infection with dermatophytes, presenting as a superficial fungal infection of the hair shafts. The hairs are broken and shortened, and the presence of scaling may mimic seborrheic dermatitis. The usually causative organisms are species of the genera Trichophyton and Microsporum. T. tonsorans is the most common cause of tinea capitis in the US. Other causes are M. audouinii, M. canis, M. ferrugineum, M. gypseum, M. violaceum, M. schoenleini, and, very rarely, T. rubrum. This report concerns a patient presenting with tinea capitis associated with Cryptococcus laurentii and Candida parapsilosis, who was subsequently discovered to have concomitant trichotillomania.

\section{Case report}

An 11-year-old boy presented on March 31, 2010 with itchy skin and a history of atopic dermatitis. Examination showed a healthy appearing male with a weight of $126 \mathrm{lb}$ and a height of $4 \mathrm{ft} 11 \mathrm{in}$, and a widespread papular eruption, with diffuse and scattered patches of alopecia on the scalp, and slight scaling (Figure 1). Based on clinical observation, the diagnosis was tinea capitis and atopic dermatitis. Bloods were drawn in anticipation of treatment with griseofulvin, and mometasone cream and ciclopirox shampoo were given for the atopic rash. The patient was seen at follow-up on April 14, 2010, with some improvement observed from treatment with ciclopirox shampoo. A hematology and biochemistry screen was normal, and the patient was started empirically on griseofulvin ultramicrosize $125 \mathrm{mg}$ three times daily. 


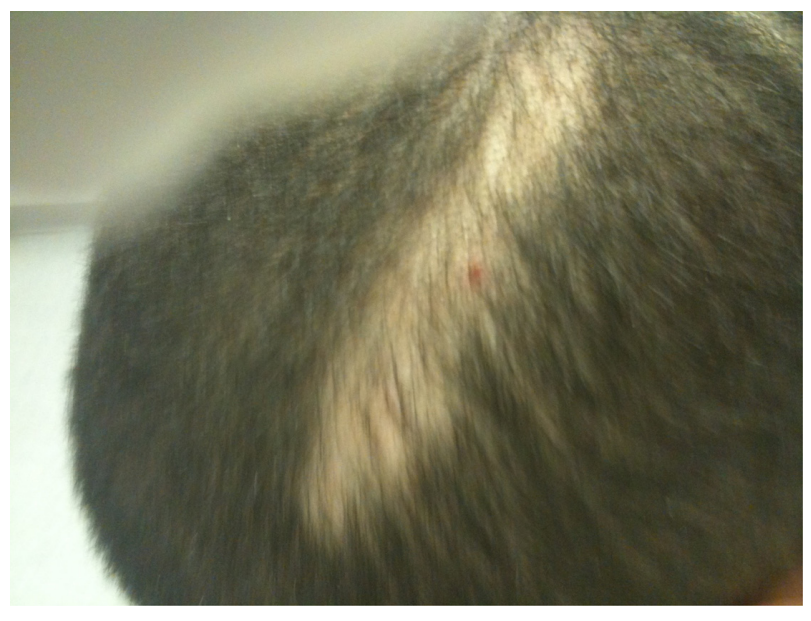

Figure I Initial presentation of alopecia on the scalp of an II-year-old boy.

The patient returned on April 27, 2010, at which time the mother had noted mild improvement, and, on May 11, 2010, she reported some hair regrowth on the medication prescribed (Figure 2). Blood tests were normal, so griseofulvin was continued. When the patient attended a further appointment on June 23,2010 , the mother was unsure if there had been any more improvement. The griseofulvin dose was increased to $250 \mathrm{mg}$ three times daily. Blood tests were normal. The patient returned on July 14, 2010, and acne papules were noted on the face and treated with clindamycin lotion. Laboratory tests were normal. Griseofulvin was continued at $250 \mathrm{mg}$ three times daily.

The patient returned for follow-up on August 4, 2010. His acne was improving on continued treatment with clindamycin gel. Moderate improvement in tinea capitis was noted. Griseofulvin $250 \mathrm{mg}$ once daily was continued. A fungal specimen was taken from the scalp and sent to the laboratory for culture, which was reported on August 19, 2010 as show-

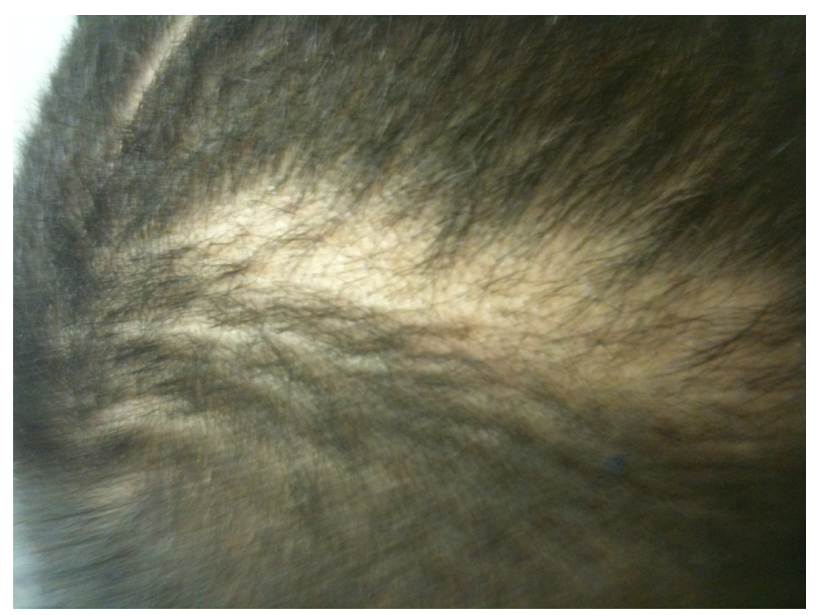

Figure 2 Regrowth noted by provider and patient's mother after 6 weeks of griseofulvin. ing C. parapsilosis. The patient returned on August 26, 2010, at which time he was started on fluconazole tablets $200 \mathrm{mg}$, two once daily, and griseofulvin was discontinued.

The patient returned on September 23, 2010, when the mother reported that the patient's scalp looked worse and with more hair falling out. Examination showed extensive areas of alopecia. The patient was continued on fluconazole, and returned on October 7, 2010, with the mother reporting mild improvement but that the patient was taking fluconazole $100 \mathrm{mg}$ instead of $200 \mathrm{mg}$. The dose was adjusted and another scalp culture was taken. The patient was seen again on October 27, 2010, with the previous culture of the scalp showing no growth, which was confirmed by the mother's observations (Figure 3). Blood tests were normal, and oxiconazole lotion was prescribed.

The patient returned on November 18, 2010, and the mother reported mild improvement in the appearance of the scalp. Eczema on the abdomen had shown mild improvement with topical steroids and emollients. Because of the negative fungal culture, the condition was considered to be resolved and more oxiconazole lotion was prescribed. The patient returned on January 13, 2011, at which time the mother reported that his hair was falling out again, and the patient himself described itching of the scalp. Another fungal culture specimen was taken from the scalp, and was reported on February 1, 2011 as showing moderate growth of $C$. laurentii. The patient was seen again on February 24, 2011 and restarted on fluconazole $400 \mathrm{mg}$ once daily. The mother reported that the tinea capitis had returned. Blood tests were normal. The attending physician made the decision to stop fluconazole on February 26, 2011, pending further workup. Oxiconazole lotion was prescribed at a follow-up visit on March 3, 2011. Blood work was normal. The patient was seen again on March 17, 2011, and prescribed

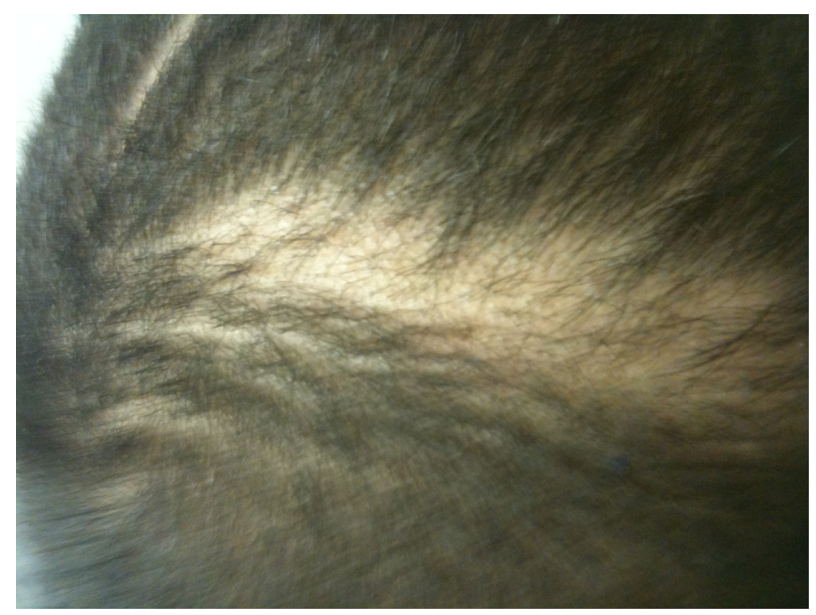

Figure 3 Patient's mother reported no further improvement in alopecia after 12 weeks of griseofulvin. 

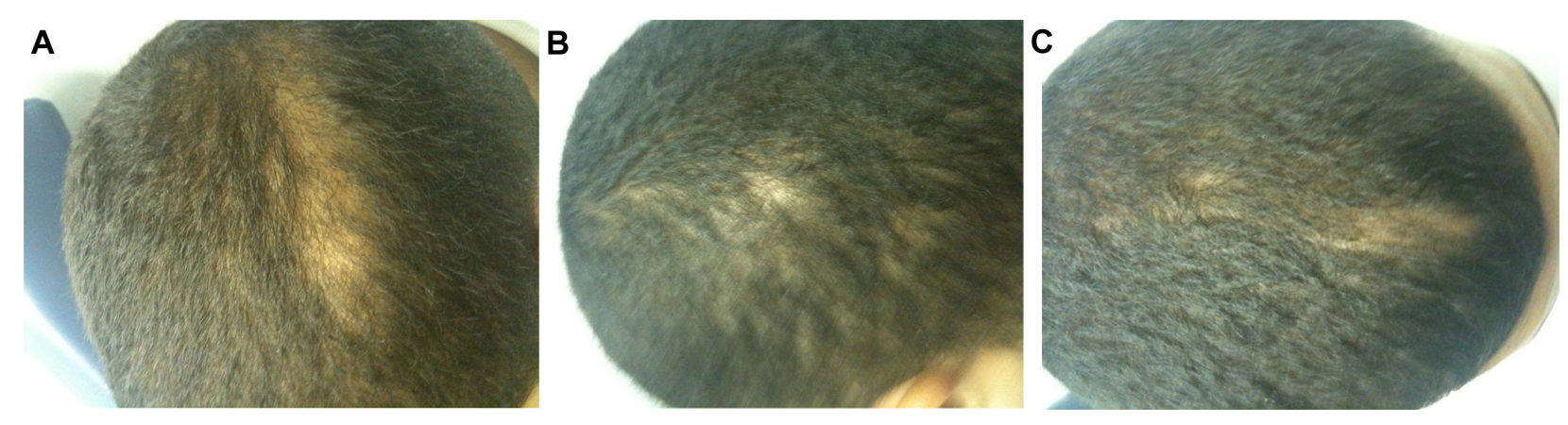

Figure 4 (A-C) Worsening of alopecia seen in three different areas on the scalp at follow-up approximately I year after initial visit.

oxiconazole cream twice daily and ketoconazole shampoo daily. Hematology and biochemistry screening continued to be normal, and continued hair loss was reported by the mother.

On March 23, 2011, extensive alopecia was noted on three areas of the scalp (Figure 4A-C), so a consultation was requested with an infectious disease consultant by one of the providers. After this consultation, the patient was started on terbinafine $250 \mathrm{mg}$ daily. Repeat culture of the scalp was done, along with testing for human immunodeficiency virus, antinuclear antibody, and protein electrophoresis.

All blood tests were within normal range. A follow-up appointment was scheduled for 1 month, at which time improvement was noted and culture was negative for fungi and bacteria. A $4 \mathrm{~mm}$ punch biopsy of an alopecic area of the scalp was sent to pathology. The report showed no organisms and was interpreted as consistent with trichotillomania. Subsequent discussion with the patient and his mother revealed that he had been pulling hairs from his head for as long as he could remember. He was referred for psychiatric evaluation, and did not return for follow-up.

\section{Discussion}

To the authors' knowledge, $C$. laurentii associated with tinea capitis has never been reported previously, although there have been rare reports of its association with skin infection. ${ }^{2}$ The organism has its reservoir in soil. It is rarely pathogenic in humans, with only 15 cases reported to produce disease. ${ }^{3}$ It is a yeast fungus found in pigeon feces, which contaminates soil, is occasionally found in vegetables or milk from infected cows, ${ }^{4}$ and is transmitted to humans through direct contact or inhaled fomites. ${ }^{5}$ Cheng et al reported a case of $C$. laurentii fungemia in a premature neonate who responded to intravenous administration of amphotericin B. ${ }^{3}$

Terbinafine is active against dermatophytes and yeasts, but less so against Candida albicans. ${ }^{6}$ However, it is highly active against $C$. parapsilosis and C. laurentii. Terbinafine is chemically an allylamine, and is used both orally and topically to treat fungal infections of the skin, nails, and hair. ${ }^{7,8}$ This patient responded well to treatment with terbinafine for the infection, but had the complicating issue of trichotillomania.

\section{Disclosure}

The author reports no conflicts of interest in this work.

\section{References}

1. Kao GK. Tinea capitis. Available from: http://emedicine.medscape.com/ article/1091351-overview. Accessed February 7, 2013.

2. Johnson LB, Bradley SF, Kauffman CA. Fungaemia due to Cryptococcus laurentii and a review of non-neoformans cryptococcaemia. 1997;47:277-280.

3. Cheng MF, Chiou CC, Liu YC, Wang HC, Hsieh KS. Cryptococcus laurentii fungemia in a premature neonate. J Clin Microbiol. 2001;39:1608-1611.

4. Chand-Goyal T, Spotts RA. Enumeration of bacterial and yeast colonists of apple fruits and identification of epiphytic yeasts on pear fruits in the Pacific Northwest United States. Microbiol Res. 1996;151:427-432.

5. Custis PH, Haller JA, de Juan E Jr. An unusual case of cryptococcal endopthalmitis. Retina. 1995;15:300-304.

6. Ryder NS. Antifungal activity and mechanism of action of terbinafine. Rev Contemp Pharmacother. 1997;8:275-287.

7. Hiratani T, AsagiY, Yamaguchi $H$. Evaluation of in vitro antimycotic activity of terbinafine, a new allylamine agent. Jpn J Med Mycol. 1991;32:323-332.

8. Villars VV, Jones TC. Special features of the clinical use or oral terbinafine in the treatment of fungal diseases. Br J Dermatol. 1992; 129 Suppl 39:61-69.
Clinical, Cosmetic and Investigational Dermatology

\section{Publish your work in this journal}

Clinical, Cosmetic and Investigational Dermatology is an international, peer-reviewed, open access, online journal that focuses on the latest clinical and experimental research in all aspects of skin disease and cosmetic interventions. All areas of dermatology wil be covered; contributions will be welcomed from all clinicians and

\section{Dovepress}

basic science researchers globally. This journal is indexed on CAS The manuscript management system is completely online and includes a very quick and fair peer-review system, which is all easy to use. Visit http://www.dovepress.com/testimonials.php to read real quotes from published authors. 